\section{KOMPASS}

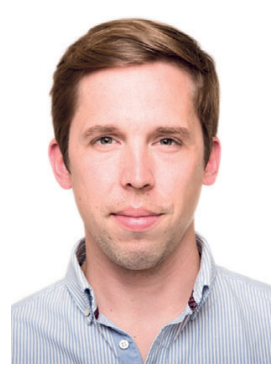

Matthias Raspe

Koordination «PneumoCampus»

\title{
Verdachtsmomente, Therapiesteckbriefe und Eigenverantwortung
}

Die Blickdiagnosen dieser Ausgabe stellen einen Patienten vor, der wegen körperlicher Abgeschlagenheit und gastrointestinalen Symptomen stationär aufgenommen wird. Die auffällige bronchoalveoläre Lavage ist ein Puzzleteil, das zur späteren seltenen Diagnose führt. Was genau dahintersteckt, erfahren Sie wie immer auf der KARGER-KOMPASS-PNEUMOLOGIE-Webseite.

Dieses Heft bildet den Auftakt zu einer kleinen Serie, die sich steckbriefartig den aktuellen Therapiemaßnahmen beim nicht kleinzelligen Lungenkarzinom (NSCLC) widmet. Sie wird in den noch ausstehenden Ausgaben des aktuellen Jahrgangs unserer Zeitschrift fortgeführt und beginnt mit den Tumorstadien I und II.

Als Abschluss der Rubrik finden Sie wieder einen «Überlebenstipp für die Weiterbildung».

\section{Blickdiagnosen \\ Welche Diagnose vermuten Sie?}

Der 37-jährige Patient wurde heimatnah zur Abklärung von körperlicher Abgeschlagenheit und gastrointestinalen Symptomen aufgenommen. Es wurde eine Influenza-B-Infektion diagnostiziert. Nebenbefundlich fielen eine Polyglobulie und eine reduzierte periphere Sauerstoffsättigung unter Raumluft auf. Das RöntgenThorax (A) zeigte bereits einen auffälligen Befund. Anamnestisch war der Patient seit 5 Monaten Nichtraucher (etwa 6 Packungsjahre). Er arbeitete als Landschafts- und Gartenbauer und hatte dort regelmäßig Kontakt zu Stein- und Betonstäuben. Ansonsten waren als Vorerkrankungen eine arterielle Hypertonie (unter Therapie mit Ramipril, Metoprolol, Indapamid) und eine Adipositas vorbestehend. Allergien waren nicht bekannt. Eine starke Dyspnoe be- stand nicht. Der Patient wurde bei Verdacht auf eine interstitielle Lungenerkrankung in unsere Klinik zur weiteren Abklärung verlegt. Hier erfolgten unter anderem eine Computertomographie des Thorax (B) und eine bronchoalveoläre Lavage (BAL; (C)).

\section{Auflösung unter: \\ www.karger.com/blickdiagnose-kkp-3-2019}

Kontaktadresse: Dr. Matthias Raspe, Medizinische Klinik mit Schwerpunkt Infektiologie und Pneumologie, Charité - Universitätsmedizin Berlin, Augustenburger Platz 1, 13353 Berlin, Deutschland, matthias. raspe@charite.de

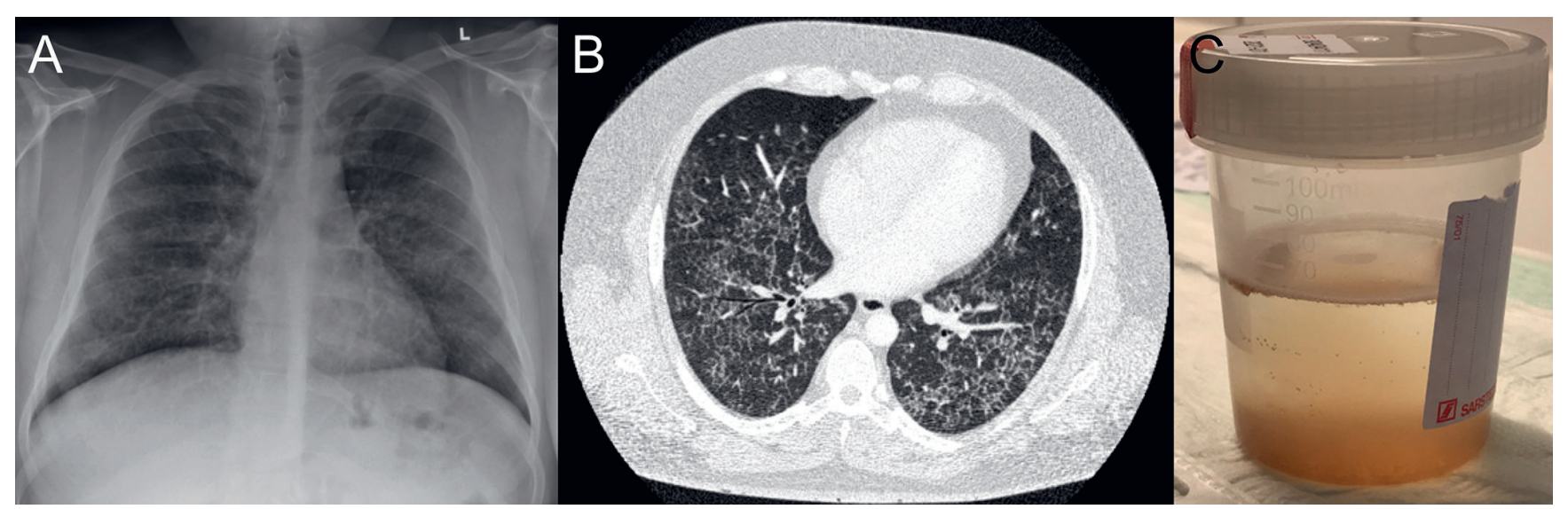

Bildbefunde (A, B) mit freundlicher Genehmigung von PD Dr. med. C. Althoff, Leitung Interventionsradiologie Charité Campus Mitte, Institut und Klinik für Radiologie CCM und CVK, Charité - Universitätsmedizin Berlin.

\section{KARGER}

(C) 2019 S. Karger GmbH, Freiburg 


\section{Steckbriefe zur aktuellen Therapie des nicht kleinzelligen Lungenkarzinoms Stadium I und II}

Das Lungenkarzinom ist in den deutschsprachigen Ländern bei Frauen der dritt- und bei Männern der zweithäufigste maligne Tumor mit einer hohen tumorspezifischen Mortalität (in Deutschland bei Männern Platz 1 und bei Frauen Platz 3). Erfreulicherweise haben sich im letzten Jahrzehnt Therapieoptionen und Prognose durch Zulassung vieler neuer Präparate aus den Bereichen Tyrosinkinaseinhibitoren und der Immuntherapie deutlich verbessert hiervon profitieren im Wesentlichen Patienten in fortgeschrittenen Krankheitsstadien.

In pneumologischen Abteilungen sind Patienten mit Lungenkarzinomen häufig. Dabei erfordert die Begleitung dieser Patienten viel Erfahrung und Wissen um den aktuellen State-of-the-art in Diagnostik und Therapie. Gerade jüngere Kolleginnen und Kollegen werden hier vor eine große Aufgabe gestellt. Mit der in dieser Ausgabe startenden 4-teiligen Serie wollen wir eine praxisnahe Übersicht zur aktuellen Therapie des nicht kleinzelligen Lungenkarzinoms (non-small cell lung carcinoma, NSCLC) geben. Im ersten Teil soll es um die Therapie des NSCLC in den Stadien I und II gehen.

Die Klassifikation des NSCLC erfolgt aktuell nach IASLC (International Association for the Study of Lung Cancer)/UICC (Union for International Cancer Control-Version 8 [1]. Die folgende Tabelle gibt einen Überblick über die Einteilung der Stadien I und II.

Die Therapie der Wahl in den lokal begrenzen Tumorstadien I und II ist eine kurativ-intendierte Operation; je nach Stadium gefolgt von einer adjuvanten Chemotherapie. Für eine Operation müssen sowohl die funktionelle als auch die technische Operabilität gegeben sein. Zur Einschätzung der funktionellen bzw. klinischen Ope-
Tumorstadien I und II des NSCLC nach IASLC/ UICC Version 8

\begin{tabular}{|c|c|c|c|}
\hline Stadium & Primärtumor & Lymphknoten & Fernmetastasen \\
\hline 0 & Tis & \multirow{6}{*}{ NO } & \multirow{8}{*}{ MO } \\
\hline$|\mathrm{A}|$ & $\begin{array}{c}\text { T1a(mi) } \\
\text { T1a }\end{array}$ & & \\
\hline $\mid A 2$ & T1b & & \\
\hline $\mathrm{IA} 3$ & $\mathrm{~T} 1 \mathrm{C}$ & & \\
\hline IB & $\mathrm{T} 2 \mathrm{a}$ & & \\
\hline$\| A$ & $\mathrm{~T} 2 \mathrm{~b}$ & & \\
\hline \multirow[t]{2}{*}{$\| B$} & $\begin{array}{l}\text { T1a-c } \\
\text { T2a } \\
\text { T2b }\end{array}$ & N1 & \\
\hline & T3 & No & \\
\hline
\end{tabular}

rabilität werden vor allem die kalkulierte postoperative Lungenfunktion und das perioperative kardiovaskuläre Risiko berücksichtigt (siehe auch offizielle Algorithmen aus den Empfehlungen der ERS (European Respiratory Society)/ESTS (European Society of Thoracic Surgeons) [2]). Für nicht operable Patienten werden durch Tumorkonferenzen/Tumorboards individuelle Therapiekonzepte festgelegt. Die folgende Abbildung zeigt den hausinternen Therapiealgorithmus der Charité - Universitätsmedizin Berlin für die Stadien I und II.

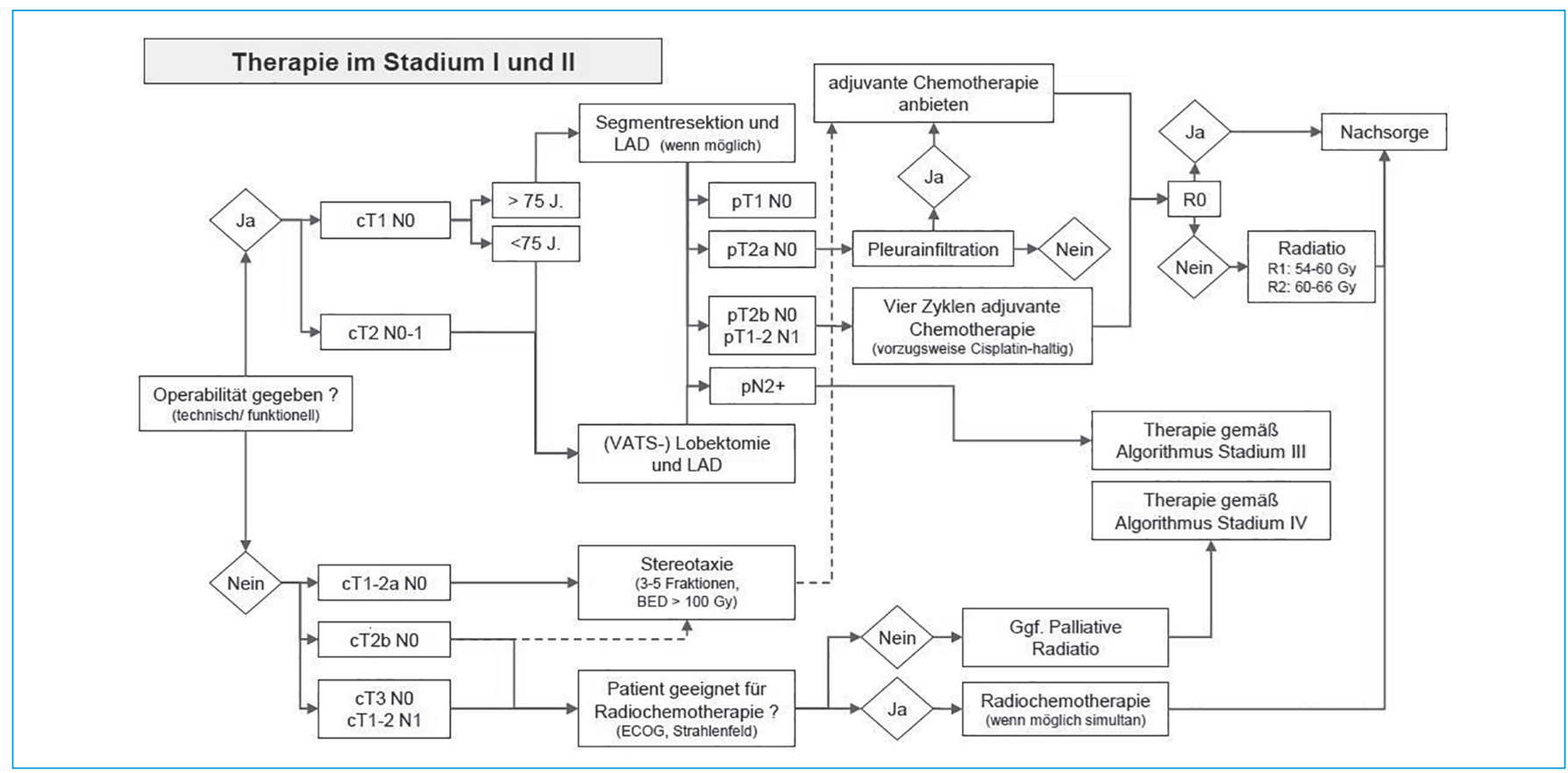

Therapiealgorithmus des NSCLC im Stadium I und II. LAD, systematische Lymphonodektomie; VATS, videoassistierte Thorakoskopie; BED, biologisch effektive Dosis. (c) Nikolaj Frost 2018. 


\section{Stadium IA und IB}

In den Stadien IA und IB ist die Operation, sofern möglich, die Therapie der Wahl. Als optimales operatives Verfahren gilt die Lobektomie mit systematischer Lymphonodektomie. Eine adjuvante Chemotherapie (Stadium IA und IB $(\leq 4 \mathrm{~cm}$ ) oder Nachbestrahlung bei R0-Situation sind nicht indiziert. Nur im Stadium IB mit einem Primarius $>4 \mathrm{~cm}$ kann eine adjuvante Chemotherapie erwogen werden, da retrospektive Analysen auf einen Vorteil der adjuvanten Therapie in dieser Situation hindeuten (eine prospektive Validierung fehlt). Weitere Situationen, bei denen bereits im Stadium I eine adjuvante Chemotherapie angeboten bzw. diskutiert werden sollte, sind eine Pleura- und/oder Lymphhämangioinvasion, da diese ebenfalls als prognostisch ungünstige Faktoren gelten. Die 5-Jahres-Überlebensraten betragen im Stadium IA zwischen 77-92\% und im Stadium IB 68\%. Ist keine Operabilität gegeben, kann eine ablative Strahlentherapie (hier vorrangig eine stereotaktische Bestrahlung) erfolgen.

\section{Stadium IIA und IIB}

Die Therapie der Wahl in den Stadien IIA und IIB ist ebenfalls, sofern keine Kontraindikationen bestehen, die Operation gefolgt von einer adjuvanten Chemotherapie. Nach einer größeren Metaanalyse wird durch die adjuvante Therapie eine Verbesserung der 5-JahresÜberlebensraten um durchschnittlich 5\% absolut erreicht [3]. Nach
UICC 8 liegen die 5-Jahres-Überlebensraten im Stadium IIA bei 60\% und im Stadium IIB bei 53\%. Als Substanzen für die adjuvante Chemotherapie ist die Kombination aus Cisplatin und Vinorelbin am besten etabliert. Für die häufig multimorbiden und betagten Patienten kann es dennoch sinnvoll sein, auf weniger toxische Substanzen/Regime wie z.B. Carboplatin und Vinorelbin/Taxan oder Splitdose-Protokolle (z.B. wöchentliche Gabe von Carboplatin/Taxan in reduzierter Dosis anstatt eines dreiwöchigen Dosisintervalls) zurückzugreifen. Eine adjuvante Strahlentherapie ist nur in Situationen mit inkompletter Resektion (R1/R2) indiziert. Die routinemäßige Anwendung von Antiangiogenesehemmern, Tyrosinkinaseinhibitoren oder Immuntherapeutika ist im adjuvanten Setting nicht etabliert.

\section{Literatur}

1 Goldstraw P, Chansky K, Crowley J, et al.: The IASLC Lung Cancer Staging Project: Proposals for Revision of the TNM Stage Groupings in the Forthcoming (Eighth) Edition of the TNM Classification for Lung Cancer. J Thorac Oncol 2016;11:39-51.

2 Brunelli A, Charloux A, Bolliger CT, et al.: ERS/ESTS clinical guidelines on fitness for radical therapy in lung cancer patients (surgery and chemo-radiotherapy). Eur Respir J 2009;34:17-41.

3 Burdett SS, Stewart LA, Rydzewska L: Chemotherapy and surgery versus surgery alone in non-small cell lung cancer. Cochrane Database Syst Rev 2007:CD006157.

\section{Kontakt:}

Dr. Matthias Raspe, Dr. Nikolaj Frost, Klinik mit Schwerpunkt Infektiologie und Pneumologie, Charité - Universitätsmedizin Berlin, Campus Virchow Klinikum, Augustenburger Platz 1, 13353 Berlin.

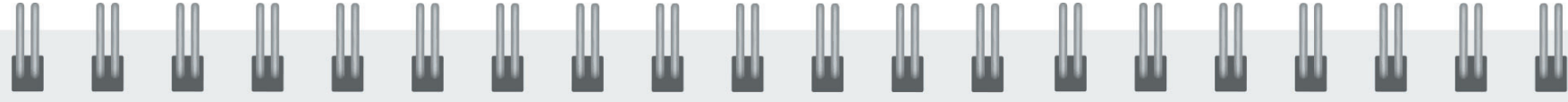

\section{Überlebenstipp für die Weiterbildung}

"Für den Erfolg der Weiterbildung tragen auch Sie als Weiterzubildende und Weiterzubildender selbst Verantwortung. So sollten Sie regelmäßige (i.d.R. jährliche) Weiterbildungsgespräche aktiv einfordern. Auch an die noch notwendigen Rotationen sollten Sie frühzeitig erinnern, damit sich die Weiterbildungszeit nicht unnötig verlängert. Denn für die Weiterbildungsbefugten ist es häufig nicht leicht, bei einer Vielzahl von betreuten Weiterbildungsassistentinnen und-assistenten den Überblick über die individuellen Bedürfnisse zu behalten.»

\section{Matthias Raspe}

